\title{
From Asia, the Novel (and Old) Fifth Malaria Plasmodium of Human Beings
}

\author{
Sergio Sabbatani ${ }^{1}$, Sirio Fiorino ${ }^{2}$ and Roberto Manfredi ${ }^{*}, 1$ \\ ${ }^{I}$ Infectious Diseases, “Alma Mater Studiorum” University of Bologna, S. Orsola-Malpighi Hospital, Bologna, Italy \\ ${ }^{2}$ Department of Internal Medicine, Budrio General Hospital, Budrio, Italy
}

\begin{abstract}
A novel form of human malaria Plasmodium knowlesi has been identified in small epidemic foci occurred during the last decade in Malaysia. Based on a review of the available literature data, the Authors underline the diagnostic importance of molecular biology examinations performed with primers which are specific of Plasmodium knowlesi, since the standard hemoscopy may fail in distinguishing Plasmodium knowlesi from Plasmodium malariae, due to their similar appearance. $P$. knowlesi has been reported as a causative parasite agent of life-threatening and even lethal forms of malaria. In humans, its clinical picture is more severe a compared to that of $P$. malariae, since the disease is characterized by a greater parasitemia, versus that is referred in the course of $P$. malariae disease. The most effective carrier of $P$. knowlesi is represented by the mosquito Anopheles leucosphyrus, which is attracted by both humans and monkeys. Among primates, the natural hosts of $P$. Knowlesi are known until now and have been represented by Macaca fascicularis and Macaca nemestina, while other monkeys including Saimiri scirea and Macaca mulatta, which cannot become infected. These might be useful in eventual experimental models. When facing the potentially severe evolution of human disease by $P$. knowlesi, we remark the key role played by a prompt disease recognition, which is expected to be more easy and obvious in patients followed in endemic countries at elevate risk, but should be carefully implemented for subjects coming back to health care services of western countries, presenting with a number of typical signs and symptoms of malaria, after travelling in South-East Asia where they were engaged in staying or making excursions in the tropical forest. In these last cases, both diagnosis and treatment should be prompt, timely, and appropriate. According to literature data, in non-severe human cases the old and trivial chloroquine remained very effective against $P$. knowlesi, achieving the disappearance of signs and symptoms in $96 \%$ of cases within the first day of pharmacological therapy. On the ground of the emerging epidemiological figures, $P$. knowlesi was added to Plasmodium vivax, Plasmodium ovale, Plasmodium malariae, and Plasmodium falciparum, as the fifth etiological agent of human malaria. During the next years, it will become mandatory to plan an adequate surveillance programme of the epidemiological evolution of this novel form of human malaria, paying also maximum attention to the clinical presentation of patients affected by $P$. knowlesi malaria, which are expected to suffer from a more severe clinical course, according to the time elapsed from the appearance of the early signs and symptoms. Some preliminary clinical figures suggest that a greater severity is related to an increased parasitemia, and parallels the increased interhuman infectious passages of parasites.
\end{abstract}

Keywords: Malaria, parasitology, Plasmodia, Plasmodium knowlesi, human health, simian-human transmission of infectious diseases, public health concerns, emerging and re-emerging infectious diseases.

\section{INTRODUCTION}

The Plasmodia parasites that are known to be involved in human malaria were four, until presently. Plasmodium vivax, Plasmodium malariae, and Plasmodium ovale, probably traced their ancient evolution by accompanying the human one: the so-called co-evolution [1-3]. On the other hand, Plasmodium falciparum is a proportionally more recent human parasite, which has been transmitted by monkeys to humans, probably during the end of Mesolithic era and the early Neolithic era [4].

Monkeys are known to be infected by over twenty different species of Plasmodia, although the primate-tohuman transmission of these parasites has been considered

*Address correspondence to this author at the Infectious Diseases, S. Orsola-Malpighi Hospital, Via Massarenti 11, I-40138 Bologna, Italy; Tel: +39-051-6363355; Fax: +39-051-343500;

E-mail: Roberto.manfredi@unibo.it since recently an infrequent event, with a reduced, related public health impact. The transmission of these Plasmodia species has been well documented, although with a varied frequency, predominantly in South-East Asia, China, and Central-Southern America [5].

The expansion of human activities in former, poorly inhabited areas (i.e. historical rain forests which were turned into production areas), and woodland regions, which are more and more frequently and explored by tourists who practice trekking activities, represent objective elements which lead to an increased risk of human Plasmodia infection, also that of simian origin. Moreover, the present demographic expansion of indigenous populations in different regions Southern Asia and Southern America, also including woodland regions or areas close to rainforest, in small villages where primary agricultural and breeding activities are performed, represent another, remarkable epidemiological risk factor. 
In the present situation, an overlapping of human and simian habitats, in the presence of mosquitoes belonging to the genus Anopheles, which are indispensable for human transmission of malaria infection, occurred.

Among twenty Plasmodia parasites which infect monkeys, five have been documented as common infectious agents for humans. They are: Plasmodium simium, Plasmodium brazilianum (in Southern America), and Plasmodium cynomolgi, Plasmodium inui, and P. knowlesi (in South-Eastern Asia) [5].

Majority of cases of simian malaria presented as a moderate, and frequently self-limiting disease in humans; an anti-malarial therapy is usually not needed, since these episodes are commonly missed or underdiagnosed. The identification of $P$. knowlesi in the states of Sarawak (Malaysian Borneo), and Sabah (another State belonging to the Malaysian Confederation), between the end of the past century and the early XXI century, all of them confirmed by molecular biology techniques [6,7], draw the attention of both researchers and clinicians who operate in South-East Asia, and are more recently by the international health care authorities, too [5]. These reports focused on patients who experienced typical malaria signs and symptoms, and received in particular a microbiological diagnosis of $P$. malariae infection. A remarkable feature of these patients was given by the frequently, elevated parasitemia, and the severity of clinical symptoms, it was this advised that these patients should refer to who did not receive an appropriate and timely treatment $[6,7]$. Four lethal cases of $P$. knowlesi human disease have been reported in the international literature, as of our knowledge [5-7].

The identification of bio-molecular primers specific of $P$. knowlesi allowed to pose a definitive diagnosis also in subjects who were parasitized, and with their erythrocytes which proved morphologically indistinguishable from those of individuals infected by $P$. malariae (but when assessed with molecular biology primers specific for $P$. malariae, failed to test positive).

The most severe clinical consequence of $P$. knowlesi malaria in human is related to the evidence of its daily replication cycle. When not counterbalanced by an adequate pharmacological therapy, $P$. knowlesi disease may rapidly reach life-threatening levels of parasitemia. We remind that the $P$. malariae cycle has its replication every three days (the so-called quartan fever), and that it never reaches a very elevated threshold of parasitized erythrocytes [6-8]. A particular feature of $P$. knowlesi is also in a similar shape of early trophozoite forms to those of $P$. falciparum, whereas all other parasite stages recall the aspect of $P$. malariae parasites.

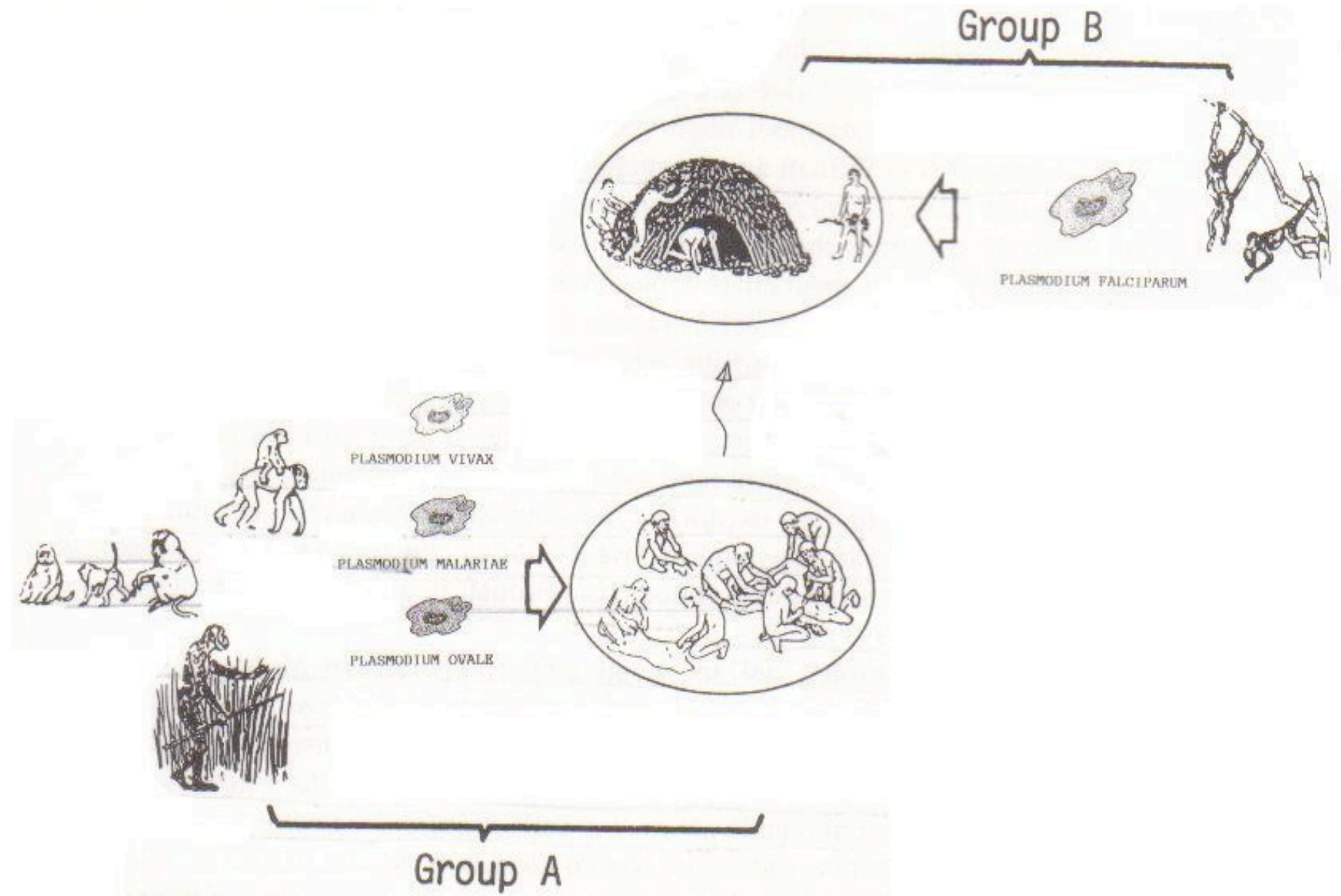

Fig. (1). The mode of evolution and transmission of Plasmodium knowlesi is shown, in parallel with that of the other four malaria Plasmodia pathogens for humans, which were known until now. 
Recent entomological studies [9] showed that the most efficient carrier of human transmission of $P$. knowlesi is the mosquito Anopheles leucosphyrus, and the most elevated sting rate is registered when humans are located close to rain forest $(6.74 \%)$, while the sting rate it is reduced inside the forest $(1.85 \%)$, and it is considerably low in the longhouses $(0.28 \%)$, which are the typical Malaysian houses.

According to the reported data, it has been speculated that humans acquire $P$. knowlesi infection inside the forest, when they go hunting, and/or when coming back to the farm on the sunset, after the working activities [10]. The monkeys which are the reservoir of $P$. knowlesi infection are Macaca nemestina and Macaca fascicularis (long-tail macaques and pig-like tail macaques). There are still a lot of doubts regarding the role of Saimiri scirea as a possible natural reservoir. Professor Babir Singh of Malaysia-Sarawak University believes that Saimiri scirea monkey may be used as an experimental model of infection, rather than representing a natural model of infection (B. Singh, Personal Communication, 2010). The monkey called Macaca mulatta, which does not have a Malaysian origin, may become incidentally infected by $P$. knowlesi, like humans. When this last monkey is infected with $P$. knowlesi, it becomes sick, and may die from this parasitic disease. This is the reason by which this monkey species may represent a reliable animal study model. Presently Macaca mulatta (Rhesus monkeys group), is rather common in Western Afghanistan, in India and Northern Thailand. In addition, historically it was more frequently retrieved in Southern China and Tibet regions, nevertheless human hunting caused its strong decline of population.

In their natural hosts, P. knowlesi causes an asymptomatic, low grade parasitemia, which is in clear contrast with hyperparasitemia followed by death in widely used experimental model of the monkey Rhesus (i.e. Macaca mulatta). As a consequence, the general appraisal is that human acquires the infection in the forest when hunting or when coming back to the farm at the sunset, after the job, while the carrier seems to acquire the Plasmodia from wild monkey; this opinion is supported by the absence of evidences of epidemic clusters inside longhouses (until now) and the most elevated number of infected mosquitoes has been registered in the rain forest or at its limits [10].

After the early reports of malaria foci in Malaysia, where the autochthonous population had been involved and the identification of anecdotal imported cases in European or Northern American tourists who spent a short time inside the rain forest, or close to South-East forests [11-15], the researches' attention towards $P$. knowlesi turned on.

In the year 2008, the outstanding Journal "Nature" first published the nuclear genomic sequency of $P$. knowlesi [16]. It was the first complete "sequencing" of a simian Plasmodium, which also makes possible a comparison with the genomic sequence of Plasmodium vivax, and that of other already sequenced Plasmodia. As opposed to other Plasmodia genomic patterns, familiar putative antigenic variants are scattered due to the genomic sequence and they are associated to repeated intrachromosomic telomers. Some of these genomic families, the so-called KIRs, contain sequences which are related to one half of the intracellular domain CD99 of the animal host, so that it was supposed that these sequences may lead to an unusual form of molecular mimicry $[16,17]$.

A general, relevant information has been provided by the recent observations and by epidemiological surveys conducted in Malaysia. The human habitat is more frequently overlapped to that of primates, and this phenomenon is mainly due to the expansion of human-driven activities (agriculture, tree reaping aimed to provide fine wood, and breeding), in areas where monkeys do live. The second emerging aspect is related to the increased presence and activity (i.e. trekking) of Western tourists, who after travelling in these inhospitable areas for a limited period of time, come back to their countries of origin [8]. In this case, the poor knowledge of simian Plasmodia and the related diagnostic problems may disorientate the attending physicians, on the basis of a standard microbiological-parasitological examination, which does not allow to distinguished Plasmodium malariae and P. knowlesi, therefore putting at risk the patient's life [8].

The molecular biology techniques prove extremely useful in depicting the general epidemiology of malaria, and to completely clarify the role and importance of mixed infections who are still largely underestimated [8]. However, we remind that this testing is expensive, and not easily accessible to all countries, with special reference to developing nations.

When taking into account the epidemiological characteristics of the areas where epidemic foci and isolated imported cases of a "travel-related" P. knowlesi disease have been registered, it does not seem possible to certainly establish whether infection was acquired by an animal reservoir (i.e. Macacus monkeys), or otherwise cases of interhuman contagion occurred. Anyway, it is now clear that these zoonoses is no more the expression of episodic facts, but it represents a true health care emergency in South-East Asia.

The co-evolutionary pathway of malaria Plasmodia together with that of the genus Homo has some shared staps. From hominids, until the contemporary humans, with the appearance of $P$. falciparum which penetrated this scenario, by infecting humans (group B) of the Mesolithic and early Neolithic eras: these humans live in simple huts at the edge of rain forest, and close to the early agriculture-devoted fields. The early introduction of the first human activities acted favourably on the spread of $P$. falciparum malaria, which is still asking a bare price, in the pathocoenosis of prehistoric populations, unavoidably conditioning the economic and anthropologic development.

With regards to the appearance of a novel malarial parasite which is epidemiologically relevant to humans, the hope is that this novel pathocoenosis remains circumscribed to some regions of South-East Asia, without the involvement of further tropical and subtropical areas of other continents. Incidentally, we remind that Afghanistan is a country where troops of Western countries are engaged in military activities, so that the surveillance is due also to the involved personnel. The diffuse presence of Macaca mulatta may represent a potential parasite reservoir for $P$. knowlesi, 
opening the way to an epidemiological spread of the infection to regions which have not been involved by this problem until now. Should this event occur, catastrophic effects are expected, not only for per the autochthonous populations who lack a specific immune memory, but also for the ever growing number of tourists who, after making their excursions in the rain forest, will be exposed to the risk of a cumbersome and displacing microbiological examination, at the time of becoming sick and coming back to the country of origin. Considerable complications related to the absence of a specific parasitological diagnosis, and eventual delays in disease recognition and management, are expected to follow.

A diagnostic retardation may become fatal, should $P$. knowlesi infection is not identified in a reasonably short time. A recent prospective clinical study conducted in the Sarawak regions of the Malaysian Borneo, disclosed that approximately $10 \%$ of patients being infected with $P$. knowlesi had severe signs and symptoms already upon presentation of briefly later, and $1-2 \%$ of cases have a fatal outcome [18]. The complications occurred in patients who survived an acute, severe $P$. knowlesi malaria attack, include the acute respiratory distress syndrome (ARDS), liver and/or kidney end-organ dysfunction, an hypotension, with or without parasitemia levels beyond 100,000 trophozoites $/ \mu \mathrm{L}$. All lethal cases of $P$. knowlesi disease were characterized by prominent abdominal signs and symptoms, associated to a combined kidney-liver dysfunction, and the concurrent already quoted hyperparasitemia [19].

From an histopathological point of view, the postmortem lesions observed in a 40-year-old man who rapidly deceased after 14 days with a "collapse"-like disease and quick death, was similar to that observed in the fatal $P$. falciparum malaria. It was the typical sequestration of pygmented, parasitized erythrocytes in the cerebellum, brain, heart, and lung vessels without any evidence of a chronic (or prolonged) inflammatory reaction in the central nervous system or other examined organs and tissues, while an extensive tubular necrosis involving the kidneys. The absence of coma and neurological features notwithstanding the petechial haemorrhage, and the parasite sequestration inside the brain, are characteristic features of lethal case reports [19].

Until now, the therapeutic perspectives seem less worrying, when a prompt diagnosis has been obtained during the early disease manifestation. In a very recent clinical study [20] performed at the Kapit Hospital (Sarawak, Malaysian Borneo), the Authors assessed the response to a treatment with chroloquine (administered at $10 \mathrm{mg} / \mathrm{Kg}$ of body weight, followed by $5 \mathrm{mg} / \mathrm{Kg}$ after 6,24 , and 48 hours), for a total of $25 \mathrm{mg} / \mathrm{kg}$. Twenty-four hours later, oral primaquine (at $15 \mathrm{mg} / \mathrm{Kg}$ of body weight), has been delivered for two consecutive days (as the gametocidal drug). All patients had non-severe, non-complicated malaria, with a parasitemia always contained at or below 100,000 trophozoites/micron. All treated subjects successfully benefited from pharmacological treatment and $96 \%$ of them had a frank improvement of signs and symptoms already evident after the first 24 hours of treatment [20]. The data presented in this Malaysian study demonstrate that conventional chloroquine dosages are associated to an initial parasite clearance which was even more rapid, as compared to that of $P$. vivax (which was used as a parasite control group in the Malaysian experience). Furthermore, this therapeutic response proved to be the most rapid, amongst all malarial forms. This last clinical experience seems very encouraging, since it demonstrates that a low-cost drug like the chloroquine may be affordable just in developing countries [20], where endemic simian-human malaria has been demonstrated to harbour. The Authors however recommend to develop further, in-depth studies, in order to assess whether this treatment may be used also in the severe forms of $P$. knowlesi human malaria.

\section{ACKNOWLEDGEMENTS}

None Declared.

\section{CONFLICT OF INTEREST}

None Declared.

\section{REFERENCES}

[1] Capasso L. L'origine delle malattie. Solfanelli, Chieti, Italy, 1985.

[2] Kim KC. Coevolution of parasitic arthropods and mammals. New York, USA: John Wiley \& Sons 1985.

[3] Cockburn A. The evolution and eradication of infectious diseases. Greenwood Press: Westport, 1963.

[4] Cohen MN, Armelagos GJ. In Paleopathology at the Originis of Agriculture. Academic Press Inc.: Orlando, USA 1984.

[5] Centers for Disease Control and Prevention (CDC). Simian malaria in a US traveler. Morbid Mortal Wkly Rep 2009; 58: 229-32.

[6] Sing B, Sung LK, Matusop A, et al. A large focus of naturally acquired Plasmodium knowlesi infections in human beings. Lancet 2004; 363: 1017-24.

[7] Cox-Singh J, Davis TME, Lee KS, et al. Plasmodium knowlesi malaria in humans is widely distributed and potentially lifethreatening. Clin Infect Dis 2008; 46: 165-71.

[8] White NJ. Plasmodium knowlesi: the fifth human malaria parasite: Editorial Commentary. Clin Infect Dis. 2008; 46: 172-3.

[9] Vythlingam I, Tan CH, Asmad M, Chan ST, Lee KS, Singh B. Natural transmission of Plasmodium knowlesi to humans by Anopheles latens in Sarawak, Malaysia. Trans R Soc Trop Med Hyg 2006; 100: 1087-1088.

[10] Tan CH, Vythlingam I, Matusop A, Chan ST, Singh B. Bionomics of Anopheles latens in Kapit, Sarawak, Malaysian Borneo in relation to the transmission of zoonotic simian malaria parasite Plasmodium knowlesi. Malaria J 2008; 7: 52-9.

[11] Kantele A, Marti H, Felger I, Müller D, Jokiranta ST. Monkey malaria in an European traveller returning from Malaysia. Emerg Infect Dis 2008; 14: 1434-6.

[12] Jongwutiwes S, Putaporntip C, Iwasatit T, Sata T, Kambara H. Naturally acquired Plasmodium knowlesi malaria in human, Thailand. Emerg Infect Dis 2004; 10: 2211-3.

[13] Zhu HM, Li J, Zheng H. Human natural infection of Plasmodium knowlesi. Zhongguo Ji Sheng Chong Xue Yu Ji Sheng Chong Bing Za Zhi 2006; 24: 70-1.

[14] Ng OT, Eong OE, Chuan LC, et al. Naturally acquired human Plasmodium knowlesi infection, Singapore. Emerg Infect Dis 2008; 14: 814-6.

[15] Bronner V, Divis PCS, Färnert A, Singh B. Swedish traveller with Plasmodium knowlesi malaria after visiting Malaysian Borneo. Malaria J 2009; 8: 1-5.

[16] Carlton JM, Adams JH, Slva JC, et al. Comparative genomics of the neglected human parasite Plasmodium vivax. Nature 2008; 455: 757-63. 
[17] Pain A, Böhme U, Berry AE, et al. The genome of the simian and human malaria parasite Plasmodium knowlesi. Nature 2008; 455: 799-803.

[18] Daneshvar C, Davis T, Cox-Singh J, et al. Clinical and laboratory features of human Plasmodium knowlesi infection. Clin Infect Dis 2009; 49: 852-60.
[19] Cox-Singh J, Hiu J, Lucas SB, et al. Severe malaria - a case of fatal Plamodium knowlesi infection with post-mortem findings: a case report. Malaria J 2010; 9:1-7

[20] Daneshvar C, Davis TME, Cox Singh J, et al. Clinical and parasitological response to oral chloroquine and primaquine in uncomplicated human Plasmodium knowlesi infections. Malaria J 2010; 9: 238:18-21.

Received: June 25, 2011

(C) Sabbatani et al.; Licensee Bentham Open.

This is an open access article licensed under the terms of the Creative Commons Attribution Non-Commercial License (http://creativecommons.org/licenses/by$\mathrm{nc} / 3.0 /$ ), which permits unrestricted, non-commercial use, distribution and reproduction in any medium, provided the work is properly cited. 